\title{
ACYCLIC 2-DIMENSIONAL COMPLEXES AND QUILLEN'S CONJECTURE
}

\author{
KEVIN IVÁN PITERMAN ${ }^{1}$, IVÁN SADOFSCHI COSTA ${ }^{2}$, \\ AND ANTONIO VIRUEL ${ }^{3}$
}

\begin{abstract}
Let $G$ be a finite group and $\mathcal{A}_{p}(G)$ be the poset of nontrivial elementary abelian $p$-subgroups of $G$. Quillen conjectured that $O_{p}(G)$ is nontrivial if $\mathcal{A}_{p}(G)$ is contractible. We prove that $O_{p}(G) \neq 1$ for any group $G$ admitting a $G$-invariant acyclic $p$-subgroup complex of dimension 2. In particular, it follows that Quillen's conjecture holds for groups of $p$-rank 3. We also apply this result to establish Quillen's conjecture for some particular groups not considered in the seminal work of Aschbacher-Smith.
\end{abstract}

\section{INTRODUCTION}

The study of the poset $\mathcal{S}_{p}(G)$ of nontrivial $p$-subgroups of a finite group $G$ started when K.S. Brown proved that the Euler characteristic $\chi\left(\mathcal{K}\left(\mathcal{S}_{p}(G)\right)\right)$ of its order complex is 1 modulo the greatest power of $p$ dividing the order of $G$ Bro75]. Recall that the order complex $\mathcal{K}(X)$ of a poset $X$ is the simplicial complex whose simplices are the finite nonempty totally ordered subsets of $X$. Some years later, D. Quillen studied the homotopy properties of $\mathcal{K}\left(\mathcal{S}_{p}(G)\right)$ Qui78. In that article, Quillen considered the subposet $\mathcal{A}_{p}(G)$ of nontrivial elementary abelian $p$-subgroups and proved that its order complex is homotopy equivalent

2010 Mathematics Subject Classification. 57S17, 20D05, 57M20, 55M20, 55M35, $57 \mathrm{M} 60$.

Key words and phrases. Quillen's conjecture, poset, $p$-subgroups.

This work was partially done at the University of Málaga, during a research stay of the first two authors, supported by project MTM2016-78647-P.

1 Supported by a CONICET doctoral fellowship and grants CONICET PIP $11220170100357 \mathrm{CO}$ and UBACyT 20020160100081BA.

2 Supported by a CONICET postdoctoral fellowship and grants ANPCyT PICT2017-2806, CONICET PIP 11220170100357CO and UBACyT 20020160100081BA.

3 Partially supported by Ministerio de Economía y Competitividad (Spain), grant MTM2016-78647-P (AEI/FEDER, UE, support included). 
to $\mathcal{K}\left(\mathcal{S}_{p}(G)\right)$ Qui78, Proposition 2.1]. Quillen also proved that, if the largest normal $p$-subgroup $O_{p}(G)$ of $G$ is nontrivial, then $\mathcal{K}\left(\mathcal{A}_{p}(G)\right)$ is contractible Qui78, Proposition 2.4] and conjectured that the converse should hold.

In this paper we study the following version of Quillen's conjecture. Recall that the homology of a poset is the homology of its order complex.

Quillen's conjecture. If $O_{p}(G)=1$ then $\widetilde{H}_{*}\left(\mathcal{A}_{p}(G)\right) \neq 0$.

Aschbacher and Smith's formulation relates rational acyclicity of $\mathcal{K}\left(\mathcal{A}_{p}(G)\right)$ with nontriviality of $O_{p}(G)$ AS93b. Thus our integral homology version is stronger than Quillen's original statement but weaker than the Aschbacher-Smith version.

Quillen proved the conjecture for solvable groups Qui78, Theorem 12.1]. In AS93b, M. Aschbacher and S.D. Smith made a huge progress on the study of this conjecture. By using the classification of finite simple groups, they proved that Quillen's conjecture holds if $p>5$ and $G$ does not contain certain unitary components. Previously, Aschbacher and Kleidman AK90] had proved Quillen's conjecture for almost simple groups (i.e. finite groups $G$ such that $L \leq G \leq \operatorname{Aut}(L)$ for some nonabelian simple group $L$ ).

The main result of our paper, which depends on the classification of the finite simple groups, is the following.

Theorem 3.2, If $X$ is an acyclic and 2-dimensional $G$-invariant subcomplex of $\mathcal{K}\left(\mathcal{S}_{p}(G)\right)$, then $O_{p}(G) \neq 1$.

Recall that the action of $G$ on $\mathcal{S}_{p}(G)$ is by conjugation. The previous result provides then a convenient tool to prove that a group verifies Quillen's conjecture.

Corollary 3.3, Let $G$ be a finite group. Suppose that $\mathcal{K}\left(\mathcal{S}_{p}(G)\right)$ admits a 2 -dimensional and $G$-invariant subcomplex homotopy equivalent to itself. Then Quillen's conjecture holds for $G$.

In particular, it follows that Quillen's conjecture holds for groups of $p$-rank 3. Recall that the $p$-rank of $G$, usually denoted by $m_{p}(G)$, is the maximum possible rank of an elementary abelian $p$-subgroup of $G$. The $p$-rank 2 case was considered by Quillen Qui78, Proposition 2.10] and is a consequence of Serre's result: an action of a finite group on a tree has a fixed point. 
In Section 4 we make an extensive use of Corollary 3.3 to establish Quillen's conjecture for some particular groups (of $p$-ranks 3 and 4) for which the hypotheses of the results of Aschbacher-Smith AS93b do not hold.

A related conjecture, due to C. Casacuberta and W. Dicks, is that a finite group acting on a contractible 2-complex has a fixed point CD92. This conjecture was studied by Aschbacher and Segev in AS93a. Posteriorly Oliver and Segev classified the groups which admit a fixed point free action on an acyclic (finite) 2-complex OS02. Our proof of Theorem 3.2 is built upon the results of OS02, which depend on the classification of finite simple groups. Theorem 3.2 can also be seen as a special case of the Casacuberta-Dicks conjecture.

Acknowledgements. We are grateful to the anonymous referee for their suggestions which greatly improved the exposition of the paper and in particular for simplifying the proofs in Examples 4.10 and 4.11 by indicating Proposition 4.9.

\section{The Results of Oliver And Segev}

In this section we review the results of OS02 needed in the proof of Theorem 3.2. By a $G$-complex we mean a $G$-CW complex. Note that the order complex of a $G$-poset is always a $G$-complex.

Definition 2.1 (OS02). A $G$-complex $X$ is essential if there is no normal subgroup $1 \neq N \triangleleft G$ such that for each $H \subseteq G$, the inclusion $X^{H N} \rightarrow X^{H}$ induces an isomorphism on integral homology.

The main results of OS02 are the following two theorems.

Theorem 2.2 ([OS02, Theorem A]). For any finite group $G$, there is an essential fixed point free 2-dimensional (finite) acyclic G-complex if and only if $G$ is isomorphic to one of the simple groups $\mathrm{PSL}_{2}\left(2^{k}\right)$ for $k \geq 2, \mathrm{PSL}_{2}(q)$ for $q \equiv \pm 3(\bmod 8)$ and $q \geq 5$, or $\mathrm{Sz}\left(2^{k}\right)$ for odd $k \geq 3$. Furthermore, the isotropy subgroups of any such $G$-complex are all solvable.

Theorem 2.3 (@S02, Theorem B]). Let $G$ be any finite group, and let $X$ be any 2-dimensional acyclic $G$-complex. Let $N$ be the subgroup generated by all normal subgroups $N^{\prime} \triangleleft G$ such that $X^{N^{\prime}} \neq \emptyset$. Then $X^{N}$ is acyclic; $X$ is essential if and only if $N=1$; and the action of $G / N$ on $X^{N}$ is essential.

The set of subgroups of $G$ will be denoted by $\mathcal{S}(G)$. 
Definition 2.4 (OS02). By a family of subgroups of $G$ we mean any subset $\mathcal{F} \subseteq \mathcal{S}(G)$ which is closed under conjugation. A nonempty family is said to be separating if it has the following three properties: (a) $G \notin \mathcal{F}$; (b) if $H^{\prime} \subseteq H$ and $H \in \mathcal{F}$ then $H^{\prime} \in \mathcal{F}$; (c) for any $H \triangleleft K \subseteq G$ with $K / H$ solvable, $K \in \mathcal{F}$ if $H \in \mathcal{F}$.

For any family $\mathcal{F}$ of subgroups of $G$, a $(G, \mathcal{F})$-complex will mean a $G$-complex all of whose isotropy subgroups lie in $\mathcal{F}$. A $(G, \mathcal{F})$-complex is $H$-universal if the fixed point set of each $H \in \mathcal{F}$ is acyclic.

Lemma 2.5 (OS02, Lemma 1.2]). Let $X$ be any 2-dimensional acyclic $G$-complex without fixed points. Let $\mathcal{F}$ be the set of subgroups $H \subseteq G$ such that $X^{H} \neq \emptyset$. Then $\mathcal{F}$ is a separating family of subgroups of $G$, and $X$ is an $H$-universal $(G, \mathcal{F})$-complex.

If $G$ is not solvable, the separating family of solvable subgroups of $G$ is denoted by $\mathcal{S} \mathcal{L} \mathcal{V}$.

Proposition 2.6 ([OS02, Proposition 6.4]). Assume that $L$ is one of the simple groups $\mathrm{PSL}_{2}(q)$ or $\mathrm{Sz}(q)$, where $q=p^{k}$ and $p$ is prime $(p=2$ in the second case). Let $G \subseteq \operatorname{Aut}(L)$ be any subgroup containing $L$, and let $\mathcal{F}$ be a separating family for $G$. Then there is a 2-dimensional acyclic $(G, \mathcal{F})$-complex if and only if $G=L, \mathcal{F}=\mathcal{S L V}$, and $q$ is a power of 2 or $q \equiv \pm 3(\bmod 8)$.

Definition 2.7 ( OS02, Definition 2.1]). For any family $\mathcal{F}$ of subgroups of $G$ define

$$
i_{\mathcal{F}}(H)=\frac{1}{\left[N_{G}(H): H\right]}\left(1-\chi\left(\mathcal{K}\left(\mathcal{F}_{>H}\right)\right)\right) .
$$

Lemma 2.8 ( $\mathrm{OS02}$, Lemma 2.3]). Fix a separating family $\mathcal{F}$, a finite $H$-universal $(G, \mathcal{F})$-complex $X$, and a subgroup $H \subseteq G$. For each $n$, let $c_{n}(H)$ denote the number of orbits of $n$-cells of type $G / H$ in $X$. Then $i_{\mathcal{F}}(H)=\sum_{n \geq 0}(-1)^{n} c_{n}(H)$.

Proposition 2.9 ( $\mathrm{OS02}$, Tables 2,3,4]). Let $G$ be one of the simple groups $\mathrm{PSL}_{2}\left(2^{k}\right)$ for $k \geq 2, \mathrm{PSL}_{2}(q)$ for $q \equiv \pm 3(\bmod 8)$ and $q \geq 5$, or $\mathrm{Sz}\left(2^{k}\right)$ for odd $k \geq 3$. Then $i_{\mathcal{S L V}}(1)=1$.

\section{The TWo-Dimensional CASE}

Using the results of Oliver and Segev stated in the previous section we prove the following.

Theorem 3.1. Every acyclic 2-dimensional $G$-complex has an orbit with normal stabilizer. 
Proof. If $X^{G} \neq \emptyset$ we are done. Otherwise, $G$ acts fixed point freely on $X$. Consider the subgroup $N$ generated by the subgroups $N^{\prime} \triangleleft G$ such that $X^{N^{\prime}} \neq \emptyset$. Clearly $N$ is normal in $G$. By Theorem $2.3 Y=X^{N}$ is acyclic (in particular it is nonempty) and the action of $G / N$ on $Y$ is essential and fixed point free. By Lemma 2.5 $\mathcal{F}=\left\{H \leq G / N: Y^{H} \neq \emptyset\right\}$ is a separating family and $Y$ is an $H$-universal $(G / N, \mathcal{F})$-complex. Thus, Theorem 2.2 asserts that $G / N$ must be one of the groups $\mathrm{PSL}_{2}\left(2^{k}\right)$ for $k \geq 2, \operatorname{PSL}_{2}(q)$ for $q \equiv \pm 3(\bmod 8)$ and $q \geq 5$, or $\operatorname{Sz}\left(2^{k}\right)$ for odd $k \geq 3$. In any case, by Proposition 2.6 we must have $\mathcal{F}=\mathcal{S} \mathcal{L} \mathcal{V}$. By Proposition

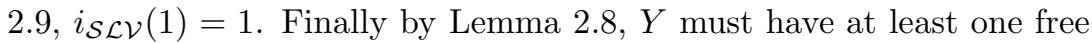
$G / N$-orbit. Therefore $X$ has a $G$-orbit of type $G / N$ and we are done.

Theorem 3.2. If $X$ is an acyclic and 2-dimensional $G$-invariant subcomplex of $\mathcal{K}\left(\mathcal{S}_{p}(G)\right)$, then $O_{p}(G) \neq 1$.

Proof. By Theorem 3.1 there is a simplex $\sigma=\left(A_{0}<\ldots<A_{j}\right)$ of $X$ with stabilizer $N \triangleleft G$. Since $A_{0} \triangleleft N$, we deduce that $O_{p}(N)$ is nontrivial. On the other hand, $N \triangleleft G$ and $O_{p}(N)$ char $N$ implies that $O_{p}(N) \triangleleft G$. Therefore $O_{p}(N) \leq O_{p}(G)$ and $O_{p}(G)$ is thus nontrivial.

From Theorem 3.2 we deduce:

Corollary 3.3. Let $G$ be a finite group. Suppose that $\mathcal{K}\left(\mathcal{S}_{p}(G)\right)$ admits a 2 -dimensional and $G$-invariant subcomplex homotopy equivalent to itself. Then Quillen's conjecture holds for $G$.

Since the $p$-rank of $G$ is equal to $\operatorname{dim} \mathcal{K}\left(\mathcal{A}_{p}(G)\right)+1$ we obtain:

Corollary 3.4. Let $G$ be a finite group of p-rank 3 . If $\widetilde{H}_{*}\left(\mathcal{A}_{p}(G)\right)=0$ then $O_{p}(G) \neq 1$.

We now apply Corollary 3.3 to obtain results for some related $p$ subgroup complexes. Recall that a $p$-subgroup $Q \leq G$ is radical if $Q=$ $O_{p}\left(N_{G}(Q)\right)$. The Bouc poset $\mathcal{B}_{p}(G)$ is the poset of nontrivial radical $p$ subgroups of $G$. It is well-known that $\mathcal{K}\left(\mathcal{B}_{p}(G)\right)$ is homotopy equivalent to $\mathcal{K}\left(\mathcal{S}_{p}(G)\right)$ Bou84. Then by Corollary 3.3 we have

Corollary 3.5. Let $G$ be a finite group such that $\mathcal{B}_{p}(G)$ has height 2 . If $\widetilde{H}_{*}\left(\mathcal{B}_{p}(G)\right)=0$ then $O_{p}(G) \neq 1$.

We say that a poset $X$ is a reduced lattice if it is obtained from a finite lattice by removing its minimum and maximum. If $X$ is a reduced lattice, $\mathfrak{i}(X)$ denotes the subposet of $X$ given by the elements which can be written as the infimum of a set of maximal elements of $X$. It is a 
general fact that the order complex of $\mathfrak{i}(X)$ is homotopy equivalent to the order complex of $X$ for any reduced lattice $X$ [Bar11, Section 9.1]. Hence by Corollary 3.3 , we have

Corollary 3.6. Let $G$ be a finite group. If either $\mathfrak{i}\left(\mathcal{S}_{p}(G)\right)$ or $\mathfrak{i}\left(\mathcal{A}_{p}(G)\right)$ has height 2, then $G$ satisfies Quillen's conjecture.

For a detailed account of the relations between the different $p$-subgroup complexes, see Smi11.

\section{Some examples}

In this section we apply the corollaries of Theorem 3.2 to establish Quillen's conjecture for some groups constructed so that the hypotheses of the results of $\mathrm{AS} 93 \mathrm{~b}$ ] are not satisfied. The main result of $\mathrm{AS} 93 \mathrm{~b}$ is the following.

Theorem 4.1 (Aschbacher-Smith AS93b, Main Theorem]). Let $G$ be a finite group and $p>5$ a prime number. Assume that whenever $G$ has a unitary component $U_{n}(q)$ with $q \equiv-1 \bmod p$ and $q$ odd, then the Quillen dimension property at $p$ holds for all p-extensions of $U_{m}\left(q^{p^{e}}\right)$ with $m \leq n$ and $e \in \mathbb{Z}$. Then $G$ satisfies Quillen's conjecture.

Recall that a group $H$ satisfies the Quillen dimension property at $p$ if $\tilde{H}_{m_{p}(H)-1}\left(\mathcal{A}_{p}(H)\right) \neq 0$. The presence of simple components of $G$ isomorphic to $L_{2}\left(2^{3}\right)$ or $U_{3}\left(2^{3}\right)$ (in the $p=3$ case) and $\mathrm{Sz}\left(2^{5}\right)$ (in the $p=5$ case) is an obstruction to extending Theorem 4.1 to $p=3$ and $p=$ 5 . The case $p=2$ is not considered in [AS93b] and would require a much more detailed analysis. One of the first steps in the proof of Theorem 4.1 is the reduction to the case $O_{p^{\prime}}(G)=1$ (see AS93b, Proposition 1.6]). To do this, AS93b, Theorems 2.3 and 2.4] are needed and these theorems make a strong use of the hypothesis $p>5$. Concretely, it is not possible to apply AS93b, Theorem 2.3] if a component of $C_{G}\left(O_{p^{\prime}}(G)\right)$ is isomorphic to $L_{2}\left(2^{3}\right), U_{3}\left(2^{3}\right)$ (if $p=3$ ) or $\mathrm{Sz}\left(2^{5}\right)$ (if $p=5$ ).

Before presenting the examples for $p=3$ and $p=5$, we give some motivation. Most of the groups $G$ in these examples satisfy the following conditions. First, $O_{p^{\prime}}(G) \neq 1$ and $C_{G}\left(O_{p^{\prime}}(G)\right)$ contains a component isomorphic to $U_{3}\left(2^{3}\right)$ if $p=3$ and to $\mathrm{Sz}\left(2^{5}\right)$ if $p=5$. Thus, we cannot find nontrivial homology for $\mathcal{A}_{p}(G)$ in the same way it is done in the proof of $\mathrm{AS93b}$, Proposition 1.6] since we are not able to invoke AS93b, Theorems 2.3 and 2.4]. Secondly, since there is an inclusion $\tilde{H}_{*}\left(\mathcal{A}_{p}\left(G / O_{p^{\prime}}(G)\right) ; \mathbb{Q}\right) \hookrightarrow \tilde{H}_{*}\left(\mathcal{A}_{p}(G) ; \mathbb{Q}\right)$ (see [AS93b, Lemma 0.12]), we 
require $O_{p}\left(G / O_{p^{\prime}}(G)\right) \neq 1$ so that $\tilde{H}_{*}\left(\mathcal{A}_{p}\left(G / O_{p^{\prime}}(G)\right)\right)=0$. Finally, we require $O_{p}(G)=1$.

The groups presented in Examples 4.5 and 4.7 have $p$-rank 3. The groups presented in Examples 4.6 and 4.8 have $p$-rank 4 and are constructed in the following way. We take a direct product of a group $N$, consisting of one or more copies of a particular simple $p^{\prime}$-group, by a group $K$ consisting of one or more copies of $L=U_{3}\left(2^{3}\right)$ if $p=3$ or $L=\mathrm{Sz}\left(2^{5}\right)$ if $p=5$. Then we take two cyclic $p$-groups $A$ and $B$ and we let them act on the direct product $N \times K$ as follows. We take a faithful action of $A \times B$ on $N$, and we choose a representation $A \times B \rightarrow \operatorname{Aut}(K)$ such that $O_{p}(K \rtimes(A \times B)) \cong O_{p}\left(C_{A}(K)\right) \neq 1$. The group $G=(N \times K) \rtimes(A \times B)$ satisfies the conditions $O_{p}(G)=1$, $O_{p^{\prime}}(G)=N \neq 1, C_{G}(N)=K$ and $O_{p}(G / N)=O_{p}(K \rtimes(A \times B)) \neq 1$. Moreover, since the $p$-rank of $L$ is at most 2 , we can construct $G$ to have $p$-rank 4 by adjusting the number of copies of $L$ in $K$.

For these groups we show that $\mathcal{K}\left(\mathcal{S}_{p}(G)\right)$ has a 2-dimensional $G$ invariant subcomplex homotopy equivalent to itself, and thus Corollary 3.3 applies.

In Examples 4.10 and 4.11 we describe two groups of 2-rank 4 such that $\mathcal{K}\left(\mathcal{S}_{2}(G)\right)$ admits a 2 -dimensional $G$-invariant homotopy equivalent subcomplex.

For the claims on the structure of the automorphism group of the finite groups of Lie type we refer to GLS98 and GLS99.

Lemma 4.2. Let $1 \rightarrow N \rightarrow G \rightarrow K \rightarrow 1$ be an extension of finite groups. Then

$$
m_{p}(G)=\max _{A \in \mathcal{S}} m_{p}\left(C_{N}(A)\right)+m_{p}(A)
$$

where $\mathcal{S}$ is the set of elementary abelian p-subgroups $1 \leq A \leq G$ such that $A \cap N=1$. In particular we have $m_{p}(G) \leq m_{p}(N)+m_{p}(K)$.

Proof. If $A \in \mathcal{S}$ we have $C_{N}(A) \times A \cong C_{N}(A) A$ and hence $m_{p}\left(C_{N}(A)\right)+$ $m_{p}(A) \leq m_{p}\left(C_{N}(A) A\right) \leq m_{p}(G)$. Taking maximum over $A \in \mathcal{S}$ gives the lower bound for $m_{p}(G)$. We now prove the other inequality. Let $E$ be an elementary abelian $p$-subgroup of $G$ and write $E=(E \cap N) A$ for some complement $A$ of $E \cap N$ in $E$. Then $m_{p}(E \cap N) \leq m_{p}\left(C_{N}(A)\right)$ and $A \in \mathcal{S}$. Now $m_{p}(E)=m_{p}(E \cap N)+m_{p}(A) \leq m_{p}\left(C_{N}(A)\right)+m_{p}(A)$, giving the upper bound for $m_{p}(G)$. For the last claim note that $C_{N}(A) \leq N$ and $m_{p}(A) \leq m_{p}(K)$ by the isomorphism theorems. 
The following lemma will be used to obtain proper subcomplexes of $\mathcal{K}\left(\mathcal{A}_{p}(G)\right)$ without changing the homotopy type. We write $X \simeq Y$ if the order complexes $\mathcal{K}(X)$ and $\mathcal{K}(Y)$ are homotopy equivalent.

Lemma 4.3. Let $G$ be a finite group and let $H \leq G$. In addition, suppose that $O_{p}\left(C_{H}(E)\right) \neq 1$ for each $E \in \mathcal{A}_{p}(G)$ with $E \cap H=1$. Then $\mathcal{A}_{p}(G) \simeq \mathcal{A}_{p}(H)$.

Proof. Consider the subposet $\mathcal{N}=\left\{E \in \mathcal{A}_{p}(G): E \cap H \neq 1\right\}$. We have order preserving maps $r: \mathcal{N} \rightarrow \mathcal{A}_{p}(H)$ and $i: \mathcal{A}_{p}(H) \hookrightarrow \mathcal{N}$ given by $r(E)=E \cap H$ and $i(E)=E$ such that $\operatorname{ir}(E) \leq E$ and $\operatorname{ri}(E)=E$. Therefore $\mathcal{N} \simeq \mathcal{A}_{p}(H)$.

Let $\mathcal{S}=\left\{E \in \mathcal{A}_{p}(G): E \cap H=1\right\}$ be the complement of $\mathcal{N}$ in $\mathcal{A}_{p}(G)$. For any $E \in \mathcal{S}$ consider $\mathcal{A}_{p}(G)_{>E} \cap \mathcal{N}=\{A \in \mathcal{N}: A>$ $E\}$. It is easy to see that $r: \mathcal{A}_{p}(G)_{>E} \cap \mathcal{N} \rightarrow \mathcal{A}_{p}\left(C_{H}(E)\right)$ defined by $r(B)=B \cap H$ is a homotopy equivalence with inverse $i(B)=B E$. Then $\mathcal{A}_{p}(G)_{>E} \cap \mathcal{N} \simeq \mathcal{A}_{p}\left(C_{H}(E)\right)$ is contractible since $O_{p}\left(C_{H}(E)\right) \neq 1$.

Now take a linear extension $E_{1}, \ldots, E_{r}$ of $\mathcal{S}$ (i.e. ennumerate the elements of $\mathcal{S}$ so that $E_{i} \leq E_{j}$ implies $\left.i \leq j\right)$ and let $X^{i}=\mathcal{N} \cup\left\{E_{1}, \ldots, E_{i}\right\}$. Note that $X^{i}=X^{i-1} \cup\left\{E_{i}\right\}$ and by the linear extension $X_{>E_{i}}^{i}=$ $\mathcal{A}_{p}(G)_{>E_{i}} \cap \mathcal{N}$, which is contractible. Now $X_{\geq E_{i}}^{i}$ is a cone over $X_{>E_{i}}^{i}$ with vertex $E_{i}$. Therefore $X^{i-1} \hookrightarrow X^{i}$ is a homotopy equivalence for each $1 \leq i \leq r$. In consequence,

$$
\mathcal{A}_{p}(G)=X^{r} \simeq X^{0}=\mathcal{N} \simeq \mathcal{A}_{p}(H) .
$$

Remark 4.4. In the above result it can be shown that if $H \triangleleft G$ then the homotopy equivalence is $G$-equivariant.

Example 4.5. Let $p=3$ and let $L=L_{2}\left(2^{3}\right) \times L_{2}\left(2^{3}\right) \times L_{2}\left(2^{3}\right)$. Let $A$ be a cyclic group of order 3 acting on $L$ by permuting the copies of $L_{2}\left(2^{3}\right)$. Take $G=L \rtimes A$. Since $m_{3}\left(L_{2}\left(2^{3}\right)\right)=1$ and $C_{L}(A) \cong L_{2}\left(2^{3}\right)$, we see that $m_{3}(G)=3$. By Corollary 3.4, $G$ satisfies Quillen's conjecture.

Example 4.6. Let $p=3, N=\mathrm{Sz}\left(2^{3}\right) \times \mathrm{Sz}\left(2^{3}\right) \times \mathrm{Sz}\left(2^{3}\right)$ and $U=U_{3}\left(2^{3}\right)$. Let $A=\langle a\rangle$ and $B=\langle b\rangle$ be cyclic groups of order 3 . We construct a semidirect product $G=(N \times U) \rtimes(A \times B)$. To do this we need to define a map $A \times B \rightarrow \operatorname{Aut}(N \times U)=\operatorname{Aut}(N) \times \operatorname{Aut}(U)$.

Choose a field automorphism $\phi \in \operatorname{Aut}\left(U_{3}\left(2^{3}\right)\right)$ of order 3 . By the properties of the $p$-group actions, there exists an inner automorphism $x \in$ $\operatorname{Inn}\left(U_{3}\left(2^{3}\right)\right)$ of order 3 commuting with $\phi$. Then $A \times B \rightarrow \operatorname{Aut}\left(U_{3}\left(2^{3}\right)\right)$ is given by $a \mapsto x$ and $b \mapsto \phi$. Choose a field automorphism $\psi \in$ $\operatorname{Aut}\left(\mathrm{Sz}\left(2^{3}\right)\right)$ of order 3 . Let $A$ act on each coordinate of $N$ as $\psi$ and 
let $B$ act on $N$ by permuting its coordinates. This gives rise to a well defined map $A \times B \rightarrow \operatorname{Aut}(N)$.

The 3-rank of $G$ is $m_{3}(G)=m_{3}\left(U_{3}\left(2^{3}\right) A B\right)$. We can take an elementary abelian subgroup $E \leq C_{U}(\phi)$ of order 9 containing $x$ since $C_{U}(\phi) \cong \mathrm{PGU}_{3}(2) \cong\left(\left(C_{3} \times C_{3}\right) \rtimes Q_{8}\right) \rtimes C_{3}$ by GLS99, Chapter 4 , Lemma 3.10] and $\mathcal{A}_{3}\left(\mathrm{PGU}_{3}(2)\right)$ is connected of height 1 . Then $E A B$ is an elementary abelian subgroup of order $3^{4}$. Hence, $m_{3}(U A B) \geq 4$. Since $m_{3}\left(U_{3}\left(2^{3}\right)\right)=2$ and $m_{3}(A B)=2$, by Lemma 4.2 we have $m_{3}(G)=4$.

By Corollary 3.3, to show that Quillen's conjecture holds for $G$ and $p=3$ it is enough to find a 2 -dimensional $G$-invariant subcomplex $X$ of $\mathcal{K}\left(\mathcal{S}_{3}(G)\right)$ homotopy equivalent to $\mathcal{K}\left(\mathcal{S}_{3}(G)\right)$ (or, equivalently, to $\left.\mathcal{K}\left(\mathcal{A}_{3}(G)\right)\right)$.

Let $H=(N \times U) \rtimes A$. Note that $H \triangleleft G$ and $m_{3}(H)=3$. Therefore, $\mathcal{K}\left(\mathcal{A}_{3}(H)\right)$ is a 2-dimensional $G$-invariant subcomplex of $\mathcal{K}\left(\mathcal{A}_{3}(G)\right)$. Now the plan is to use Lemma 4.3 to show that $\mathcal{A}_{3}(H) \simeq \mathcal{A}_{3}(G)$. Let $E \in$ $\mathcal{A}_{3}(G)$ be such that $E \cap H=1$. Then $E \cong E H / H \leq B \cong C_{3}$ and hence, $E$ is cyclic generated by some element $e \in E$. Write $e=n u a^{i} b^{j}$ with $n \in N, u \in U$ and $i, j \in\{0,1,2\}$. Note that $j \neq 0$ since $E \cap H=1$. If $v \in U$, then

$$
v^{e}=v^{n u a^{i} b^{j}}=\left(v^{u a^{i}}\right)^{b^{j}} .
$$

Since $j \neq 0$ and $e$ induces an automorphism of $U$ of order $3 \operatorname{in} \operatorname{Inn}(U) \phi^{j}$, by [GLS98, Proposition 4.9.1] and the definition of field automorphisms GLS98, Definition 2.5.13], $e$ is Inndiag $(U)$-conjugate to $\phi^{j}$ and acts as a field automorphism on $U$. In particular, $C_{U}(E)=C_{U}(e) \cong C_{U}\left(\phi^{j}\right)=$ $C_{U}(\phi)$. Note that $O_{3}\left(C_{U}(E)\right) \cong O_{3}\left(C_{U}(\phi)\right) \cong C_{3} \times C_{3} \neq 1$. Since $C_{U}(E) \triangleleft C_{H}(E)$ and $O_{3}\left(C_{U}(E)\right) \neq 1$, we conclude that $O_{3}\left(C_{H}(E)\right) \neq$ 1. By Lemma 4.3, $\mathcal{A}_{3}(G) \simeq \mathcal{A}_{3}(H)$, which is 2-dimensional and $G$ invariant. In conclusion, the subcomplex $\mathcal{K}\left(\mathcal{A}_{3}(H)\right)$ satisfies the hypothesis of Corollary 3.3 and therefore, Quillen's conjecture holds for G.

Note that $O_{3}(G)=1, O_{3^{\prime}}(G)=N, C_{G}\left(O_{3^{\prime}}(G)\right)=U_{3}\left(2^{3}\right)$ and $O_{3}\left(G / O_{3^{\prime}}(G)\right)=O_{3}\left(U_{3}\left(2^{3}\right) A B\right)=\left\langle a x^{-1}\right\rangle \cong C_{3}$.

Example 4.7. Let $p=5$. Let $r$ be a prime number such that $r \equiv 2$ or $3 \bmod 5$ and let $q=r^{5^{n}}$ with $n \geq 2$. Let $N$ be one of the simple groups $L_{2}(q), G_{2}(q),{ }^{3} D_{4}\left(q^{3}\right)$ or ${ }^{2} G_{2}\left(3^{5^{n}}\right)$ and let $A=\langle a\rangle$ be a cyclic group of order $5^{n}$. Note that $5 \nmid|N|$. Let $a$ act on $N$ as a field automorphism of order $5^{n}$. Choose a field automorphism $\phi \in \operatorname{Aut}\left(\operatorname{Sz}\left(2^{5}\right)\right)$ of order 5 and let $A$ act on $\mathrm{Sz}\left(2^{5}\right) \times \mathrm{Sz}\left(2^{5}\right)$ as $\phi \times \phi$. Now consider the semidirect product $G=\left(N \times \mathrm{Sz}\left(2^{5}\right) \times \mathrm{Sz}\left(2^{5}\right)\right) \rtimes A$ defined by this action. 
Since the Sylow 5-subgroups of $\mathrm{Sz}\left(2^{5}\right)$ are cyclic of order 25, by Lemma 4.2 we have that $m_{5}(G)=3$. By Corollary 3.4, Quillen's conjecture holds for $G$.

Moreover, $O_{5}(G)=1, O_{5^{\prime}}(G)=N, C_{G}\left(O_{5^{\prime}}(G)\right)=\operatorname{Sz}\left(2^{5}\right)^{2}$ and $O_{5}\left(G / O_{5^{\prime}}(G)\right)=C_{A}\left(\mathrm{Sz}\left(2^{5}\right)^{2}\right)=\left\langle a^{5}\right\rangle \neq 1$.

Example 4.8. Let $p=5$ and let $N=L^{5}$, where $L$ is one of the simple $5^{\prime}$-groups of the previous example. Let $A=\langle a\rangle \cong C_{5^{n}}$ and $B=\langle b\rangle \cong C_{5}$. Let $G=\left(N \times \mathrm{Sz}\left(2^{5}\right)^{2}\right) \rtimes(A \times B)$, where $a$ acts on each copy of $L$ as a field automorphism of order $5^{n}$ and trivially on $\mathrm{Sz}\left(2^{5}\right)^{2}$, and $b$ permutes the copies of $L$ and acts as a field automorphism of order 5 on each copy of $\mathrm{Sz}\left(2^{5}\right)$.

To compute the 5 -rank of $G$ we use Lemma 4.2 ,

$$
\begin{aligned}
m_{5}(G) & =m_{5}\left(\mathrm{Sz}\left(2^{5}\right)^{2} \rtimes(A \times B)\right) \\
& =m_{5}\left(A \times\left(\mathrm{Sz}\left(2^{5}\right)^{2} \rtimes B\right)\right) \\
& =m_{5}(A)+m_{5}\left(\mathrm{Sz}\left(2^{5}\right)^{2} \rtimes B\right) \\
& =1+3 \\
& =4 .
\end{aligned}
$$

Now the aim is to apply Corollary 3.3 on $G$ by finding a 2-dimensional $G$-invariant homotopy equivalent subcomplex $X$ of $\mathcal{K}\left(\mathcal{S}_{5}(G)\right)$.

Let $H=\left(N \times \mathrm{Sz}\left(2^{5}\right)^{2}\right) \rtimes A=N A \times \mathrm{Sz}\left(2^{5}\right)^{2}$. Note that $H \triangleleft G$ and $m_{5}(H)=3$. Hence $\mathcal{K}\left(\mathcal{A}_{5}(H)\right)$ is 2 -dimensional and $G$-invariant. We will show that $\mathcal{A}_{5}(H) \simeq \mathcal{A}_{5}(G)$ by applying Lemma 4.3.

Let $E \in \mathcal{A}_{5}(G)$ be such that $E \cap H=1$. Then $E$ is cyclic generated by an element $e$ of order 5 and $e=l s a^{i} b^{j}$ with $l \in N, s \in \operatorname{Sz}\left(2^{5}\right)^{2}$, $0 \leq i \leq 5^{n}-1$ and $j \in\{1,2,3,4\}$. Thus $E$ acts by field automorphisms on each copy of the Suzuki group and $e$ is $\operatorname{Inndiag}\left(\operatorname{Sz}\left(2^{5}\right)\right)$-conjugate to the field automorphism induced by $b^{j}$ on $\mathrm{Sz}\left(2^{5}\right)$ (see GLS98, Proposition 4.9.1] and Example 4.6). Hence, $C_{H}(E)=C_{N A}(E) \times C_{\mathrm{Sz}\left(2^{5}\right)^{2}}(E)$. Note that $C_{\mathrm{Sz}\left(2^{5}\right)^{2}}(E) \triangleleft C_{H}(E)$ and $C_{\mathrm{Sz}\left(2^{5}\right)^{2}}(E) \cong C_{\mathrm{Sz}\left(2^{5}\right)}(E)^{2} \cong\left(C_{5} \rtimes C_{4}\right)^{2}$ has a nontrivial normal 5-subgroup. Therefore $\mathcal{A}_{5}(G) \simeq \mathcal{A}_{5}(H)$ by Lemma 4.3 and Quillen's conjecture holds for $G$ by Corollary 3.3 applied to the subcomplex $\mathcal{K}\left(\mathcal{A}_{5}(H)\right)$.

Note that $O_{5^{\prime}}(G)=N$ and $C_{G}\left(O_{5^{\prime}}(G)\right)=\mathrm{Sz}\left(2^{5}\right)^{2}$. On the other hand, $O_{5}(G)=1$ and $O_{5}\left(G / O_{5^{\prime}}(G)\right)=A \neq 1$.

We conclude with two examples of groups satisfying Quillen's conjecture for $p=2$. We say that a finite group $G$ has the trivial intersection 
property at $p$ if any two different Sylow $p$-subgroups of $G$ have trivial intersection.

Proposition 4.9. Let $L_{1}$ and $L_{2}$ be two finite groups with the trivial intersection property at $p$. Let $L=L_{1} \times L_{2}$ and take an extension $G$ of $L$ such that $|G: L|=p$. Then $\mathfrak{i}\left(\mathcal{S}_{p}(G)\right)$ and $\mathcal{B}_{p}(G)$ are at most 2 dimensional. If in addition the Sylow p-subgroups of $L_{1}$ and $L_{2}$ have abelian $\Omega_{1}$, then $\mathfrak{i}\left(\mathcal{A}_{p}(G)\right)$ is at most 2-dimensional.

Proof. The elements of $i\left(\mathcal{S}_{p}(L)\right)$ are of the form $P_{1} \times P_{2}, 1 \times P_{2}$ or $P_{1} \times 1$, where $P_{i} \leq L_{i}$ are Sylow $p$-subgroups. Hence, $\mathfrak{i}\left(\mathcal{S}_{p}(L)\right)$ is 1-dimensional.

Now suppose that $Q_{0}<Q_{1}<\ldots<Q_{n}$ is a chain in $\mathfrak{i}\left(\mathcal{S}_{p}(G)\right)$. Then

$$
Q_{0} \cap L \leq Q_{1} \cap L \leq \ldots \leq Q_{n} \cap L
$$

is a chain in $\mathfrak{i}\left(\mathcal{S}_{p}(L)\right)$. We claim that there is at most one index $i$ such that $Q_{i} \cap L=Q_{i+1} \cap L$. To see this note that

$$
\left|Q_{j}: Q_{j} \cap L\right|=\left\{\begin{array}{ll}
1 & \text { if } Q_{j} \subseteq L \\
p & \text { if } Q_{j} \not \subset L
\end{array} .\right.
$$

We have $\left|Q_{i+1}: Q_{i}\right| \cdot\left|Q_{i}: Q_{i} \cap L\right|=\left|Q_{i+1}: Q_{i+1} \cap L\right| \cdot\left|Q_{i+1} \cap L: Q_{i} \cap L\right|$. Then if $Q_{i} \cap L=Q_{i+1} \cap L$, since $\left|Q_{i+1}: Q_{i}\right| \geq p$ we must have $\mid Q_{i}$ : $Q_{i} \cap L \mid=1$ and $\left|Q_{i+1}: Q_{i+1} \cap L\right|=p$. Then $i=\max \left\{j: Q_{j} \subseteq L\right\}$.

From this we conclude that $\operatorname{dim} \mathfrak{i}\left(\mathcal{S}_{p}(G)\right) \leq 1+\operatorname{dim} \mathfrak{i}\left(\mathcal{S}_{p}(L)\right)=2$. It is well-known that $\mathcal{B}_{p}(G)$ is a subposet of $\mathfrak{i}\left(\mathcal{S}_{p}(G)\right)$ (i.e. every radical $p$-subgroup is an intersection of Sylow $p$-subgroups). Then $\mathcal{B}_{p}(G)$ is at most 2-dimensional also. The same proof can be easily adapted to prove that, if the Sylow $p$-subgroups of $L_{1}$ and $L_{2}$ have abelian $\Omega_{1}, \mathfrak{i}\left(\mathcal{A}_{p}(G)\right)$ is at most 2-dimensional.

In the following examples we use the fact that the groups $A_{5}$ and $U_{3}\left(2^{2}\right)$ have the trivial intersection property at 2 and that $\Omega_{1}(P)$ is abelian for $P$ a Sylow 2-subgroup of either $A_{5}$ or $U_{3}\left(2^{2}\right)$.

Example 4.10. Let $G$ be the group extension $\left(A_{5} \times A_{5}\right) \rtimes C_{2}$ where the generator of $C_{2}$ acts on each coordinate as conjugation by the transposition (12). Since $m_{2}\left(A_{5}\right)=2=m_{2}\left(\operatorname{Aut}\left(A_{5}\right)\right)$, by Lemma 4.2, $G$ has 2-rank 4. By Proposition 4.9, $\mathfrak{i}\left(\mathcal{A}_{2}(G)\right), \mathfrak{i}\left(\mathcal{S}_{2}(G)\right)$ and $\mathcal{B}_{2}(G)$ are 2dimensional and then Quillen's conjecture holds for $G$ since Corollaries 3.5 and 3.6 apply.

Example 4.11. Let $G=\left(U_{3}\left(2^{2}\right) \times A_{5}\right) \rtimes C_{2}$ be the semidirect product constructed in the following way. Let $H=U_{3}\left(2^{2}\right) \times A_{5}$. Then 
$\operatorname{Out}(H) \cong \operatorname{Aut}\left(U_{3}\left(2^{2}\right)\right) / \operatorname{Inn}\left(U_{3}\left(2^{2}\right)\right) \times \operatorname{Aut}\left(A_{5}\right) / \operatorname{Inn}\left(A_{5}\right) \cong C_{4} \times C_{2}$. Take $t \in \operatorname{Out}(H)$ to be the involution which acts nontrivially on both factors. Therefore $G=H \rtimes\langle t\rangle$. Since $m_{2}\left(U_{3}\left(2^{2}\right)\right)=2=m_{2}\left(A_{5}\right)=m_{2}\left(\operatorname{Aut}\left(A_{5}\right)\right)$ and $m_{2}\left(\operatorname{Aut}\left(U_{3}\left(2^{2}\right)\right)\right)=3$, by Lemma $4.2 G$ has 2 -rank 4 . Just as before, Quillen's conjecture holds for $G$.

\section{REFERENCES}

[AK90] Michael Aschbacher and Peter B. Kleidman. On a conjecture of Quillen and a lemma of Robinson. Arch. Math. (Basel), 55(3):209-217, 1990.

[AS93a] Michael Aschbacher and Yoav Segev. A fixed point theorem for groups acting on finite 2-dimensional acyclic simplicial complexes. Proc. London Math. Soc. (3), 67(2):329-354, 1993.

[AS93b] Michael Aschbacher and Stephen D. Smith. On Quillen's conjecture for the p-groups complex. Ann. of Math. (2), 137(3):473-529, 1993.

[Bar11] Jonathan A. Barmak. Algebraic topology of finite topological spaces and applications, volume 2032 of Lecture Notes in Mathematics. Springer, Heidelberg, 2011.

[Bou84] Serge Bouc. Homologie de certains ensembles ordonnés. C. R. Acad. Sci. Paris Sér. I Math., 299(2):49-52, 1984.

[Bro75] Kenneth S. Brown. Euler characteristics of groups: the $p$-fractional part. Invent. Math., 29(1):1-5, 1975.

[CD92] Carles Casacuberta and Warren Dicks. On finite groups acting on acyclic complexes of dimension two. Publ. Mat., 36(2A):463-466 (1993), 1992.

[GLS98] Daniel Gorenstein, Richard Lyons, and Ronald Solomon. The Classification of the Finite Simple Groups. Number 3. Part I. Chapter A: Almost simple $\mathcal{K}$-groups, volume 40 of Mathematical Surveys and Monographs. American Mathematical Society, Providence, RI, 1998.

[GLS99] Daniel Gorenstein, Richard Lyons, and Ronald Solomon. The Classification of the Finite Simple Groups, Number 4. Part II, Chapters 1-4: Uniqueness Theorems, volume 40 of Mathematical Surveys and Monographs. American Mathematical Society, Providence, RI, 1999. General group theory.

[OS02] Bob Oliver and Yoav Segev. Fixed point free actions on Z-acyclic 2complexes. Acta Math., 189(2):203-285, 2002.

[Qui78] Daniel Quillen. Homotopy properties of the poset of nontrivial $p$-subgroups of a group. Adv. in Math., 28(2):101-128, 1978.

[Smi11] Stephen D. Smith. Subgroup complexes, volume 179 of Mathematical Surveys and Monographs. American Mathematical Society, Providence, RI, 2011.

Universidad de Buenos Aires. Facultad de Ciencias Exactas y Naturales. Departamento de Matemática. Buenos Aires, Argentina.

CONiCET-Universidad de Buenos Aires. Instituto de Investigaciones Matemáticas Luis A. Santaló (IMAS). Buenos Aires, Argentina. 
ACYCLIC 2-DIMENSIONAL COMPLEXES AND QUILLEN'S CONJECTURE 13

Departamento de Álgebra, Geometría y Topología, Universidad de Málaga, Campus de Teatinos, 29071 Málaga, Spain.

Email address: kpiterman@dm.uba.ar

Email address: isadof schi@dm.uba.ar

Email address: viruel@uma.es 